\title{
Research of Prognostic Role of MSI in Endometrioid Adenocarci- noma Cases That Received Postoperative Radiotherapy
}

\author{
Kadriye Sahinturk, $M D^{1}$, Candan Demiroz Abakay, $M D^{2^{*}}$, Serdar Sahinturk, MD, PhD , \\ Sebnem Ozemri Sag ${ }^{4}$, Fatma Atalay ${ }^{5}$, Fatma E Can 6 , Meral Kurt, MD², and Sibel Kahraman \\ Cetintas, $M D^{2}$
}

\begin{abstract}
${ }^{1}$ Specialist, Radiation Oncology Department, Faculty of Medicine, Uludag University, Gorukle/Bursa, Turkey ${ }^{2}$ Associate Professor, Radiation Oncology Department, Faculty of Medicine, Uludag University, Gorukle/Bursa, Turkey ${ }^{3}$ Department of Physiology, Faculty of Medicine, Uludag University, Gorukle/Bursa, Turkey ${ }^{4}$ Assistant Professor, Department of Genetics, Faculty of Medicine, Uludag University, Gorukle/Bursa, Turkey ${ }^{5}$ Associate Professor, Pathology Department, Faculty of Medicine, Uludag University, Gorukle/Bursa, Turkey ${ }^{6}$ Research Assistant, Department of Biostatics, Faculty of Medicine, Uludag University, Gorukle/Bursa, Turkey
\end{abstract}

*Corresponding author: Candan Demiroz Abakay, MD, Associate Professor, Radiation Oncology Department, Faculty of Medicine, Uludag University, Gorukle/Bursa, Turkey, Tel: +905-336-663-507

\begin{abstract}
In this study, the relationship between MSI (microsatellite instability) and the parameters such as age, stage, histologic grade, recurrence, overall survival (OS), disease free survival (DFS) were investigated in 99 endometrioid adenocancer patients. Our aim is to find out the whether there is a prognostic value of $\mathrm{MSI}$ in endometrium adenocarcinoma. In MSI analysis, by employing the Promega MSI Analysis System. 'IBM SPSS v.20' was used for statistical analysis. MSI was identified in $19.1 \%$ of patients. There was no statistically significant effect of MSI on OS ( $p=0.404)$ or on DFS ( $p=0.407$ ). MSI was found associated with younger age $(p=0.032)$, lymph node involvement $(p=0.012)$ and advanced stage $(p=0.014)$.
\end{abstract}

\section{Keywords}

Endometrium cancer, Microsatellite instability, Radiotherapy

\section{Introduction}

Endometrium cancer (EC) is the most common malignancy of the female genital tract $[1,2]$. The recurrence rate is $4-19 \%$ in the studies that performed with different risk groups (high risk stage I and stage II patients) $(136,137)$. Also $70-80 \%$ of the recurrences happen in the first three years $(139,140,141)$.
Risk stratification based on clinicopathologic criteria and the use of adjuvant treatments seems to be unsuccessful. Therefore, molecular characterization represents an attractive alternative that could also be effective in the evaluation of prognosis. MSI is a common genetic alteration in EC [3] (135). Epigenetic alterations and mutations in the genes that codes the proteins in mismatch repair (MMR) system reflect as $\mathrm{MSI}$ in cellular phenotype. MSI is seen in $75 \%$ of familial EC and $25-30 \%$ of sporadic EC $[4,5]$.

In this study, our aim is to determine the importance of MSI in prognosis, diagnosis and treatment of endometrium adenocancer.

\section{Materials and Methods}

\section{Cases}

One hundred twenty four endometrium cancer patients who were referred for postoperative external radiotherapy and/or brachytherapy during the period between January 2002 and December 2012 at Uludag University Radiation Oncology Department. Out of three all patients received radiotherapy. Since 11 had mixed type pathology, 9 had unspecified paraffine blocks and

Citation: Sahinturk K, Abakay CD, Sahinturk S, Sag SO, Atalay F, Can FE, et al. (2019) Research of Prognostic Role of MSI in Endometrioid Adenocarcinoma Cases That Received Postoperative Radiotherapy. Int J Cancer Clin Res 6:128. doi.org/10.23937/2378-3419/1410128

Accepted: November 09, 2019; Published: November 11, 2019

Copyright: (c) 2019 Sahinturk K, et al. This is an open-access article distributed under the terms of the Creative Commons Attribution License, which permits unrestricted use, distribution, and reproduction in any medium, provided the original author and source are credited. 
2 had unsuccessful MSI analysis, study was performed with 99 patients and all re-staged according to FIGO 2009 staging system.

\section{DNA extraction and $\mathrm{MSI}$ analysis}

Tumor tissue specimens were extracted within formalin fixed, paraffin-embedded sample tissue. Tumor tissue DNA was purified by organic extraction. "QIAamp DNA FFPE Tissue; Oiagen kit" was used for DNA isolation. MSI analysis was performed by employing the Promega MSI Analysis System. Applied Biosystems 3130 Genetic Analyzer was used to separate the products obtained from fluorescently labeled polymerase chain reaction, and the data were analyzed using GeneMapper program (Applied Biosystems). This system uses 5 mononucleotide markers to identify MSI in a tumor and normal tissue DNA (BAT-25, BAT-26, NR-21, NR-24, and MONO27), and 2 pentanucleotide markers (Penta $C$ and Penta D) to identify whether the tumor and normal DNA specimens are from the same patient. Tumors were classified using the following method: If more than 2 out of 5 markers demonstrated size alterations or shifts in the tumor DNA with respect to the normal tissue DNA, this cancer was identified as MSI-high (MSI-H). Tumors with only one marker showing instability were classified as MSI-low (MSI-L) while tumors with none of the markers showing instability was classified as microsatellite stable (MSS).

\section{Statistical analysis}

Independent sample t-test was used for the data showing normal distribution and Mann-Whitney $U$ test was used for the data showing non-normal distribution. Pearson Chi-square test, Fisher exact test and Fisher Freeman Halton test were used to compare categorical variables between groups. Kaplan-Meier method was used to estimate survival. Binary logistic regression analysis was used to investigate risk factors for categorical variables. $\mathrm{P}<0.05$ indicated a significant statistical difference. All statistical analyses were performed by IBM SPSS v.20.

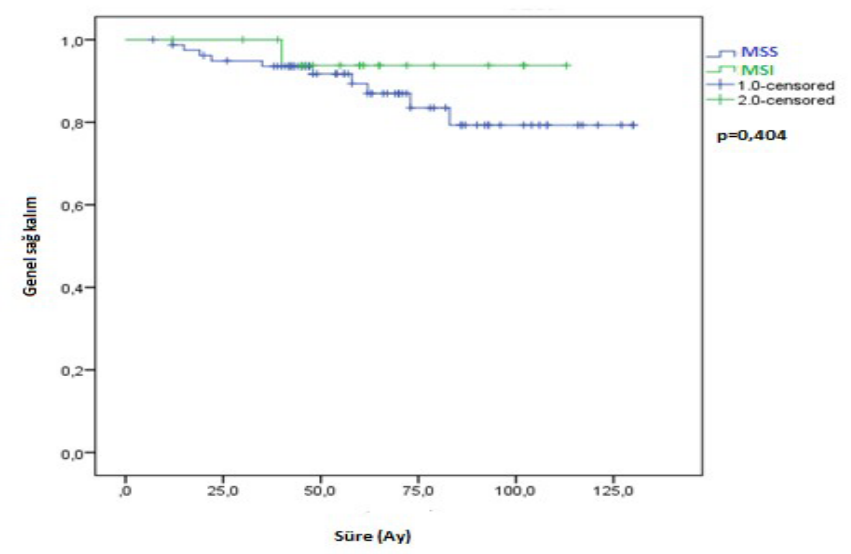

\section{Results}

Demographics and clinical characteristics are presented in Table 1. The mean age at the diagnosis was 62.7 ( \pm 9.32 ) years. 28 patients were treated with external radiotherapy and brachytherapy, 70 were treated only with brachytherapy and one who had vaginal stenosis was just treated with external radiotherapy. Thirteen patients also had chemotherapy. The recurrence ratio was $12 \%$ in total; 12 ( 3 in vaginal cuff, 3 in pelvic lymph nodes, 1 in paraaortic lymph nodes, 1 in pelvic bone, 1 in lung and 2 in peritoneum). In the last follow up, 11 patients were lost to follow up and 4 of them were found to be related to their disease. OS was $116 \pm$ 3.9 months; DFS was $115.4 \pm 3.7$ months

19 patients were MSI-H and 3 patients were MSI-L. In statistical analysis, MSI-L patients are evaluated with in MSS group. The relation between MSI status and clinicopathologic features of patients is presented in Table 2.

Table 1: Demographic and clinical characteristics of patients.

\begin{tabular}{|l|l|l|l|}
\hline Age & \multicolumn{3}{|c|}{ Adnexal invasion } \\
\hline$\leq 60$ & $38.4 \%$ & No & $93.9 \%$ \\
\hline$>60$ & $61.6 \%$ & Yes & $6.1 \%$ \\
\hline Stage & \multicolumn{3}{|c|}{ Metastatic lymph node } \\
\hline I & $40.4 \%$ & \multicolumn{2}{|c|}{} \\
\hline II & $41.4 \%$ & No & $72.7 \%$ \\
\hline III & $16.2 \%$ & Yes & $14.1 \%$ \\
\hline IV & $2 \%$ & \multicolumn{2}{|l|}{} \\
\hline Grade & & Lympho vascular invasion \\
\hline 1 & $26.5 \%$ & No & $66.6 \%$ \\
\hline 2 & $45.9 \%$ & Yes & $32.3 \%$ \\
\hline 3 & $27.6 \%$ & \multicolumn{2}{|l|}{} \\
\hline & & Myometrial invasion & $1 \%$ \\
\hline & & No & $50.5 \%$ \\
\hline & & $<1 / 2$ & $48.5 \%$ \\
\hline & & $\geq 1 / 2$ &
\end{tabular}

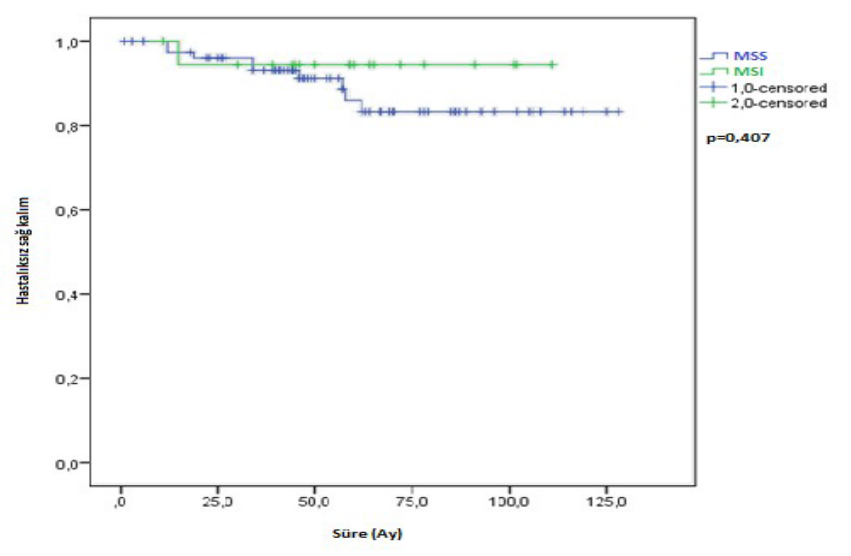

Figure 1: Kaplan-Meier curves for OS and DFS according to MSI status of patients. 
No statistically significant difference was found in OS between patients with MSS and MSI ( $p=0.404)$. The mean of OS was 108.43 months in MSI group and 114.16 months in MSS group. Also DFS was not statistically significant $(p=0.407)$. Even both of OS and DFS were better in MSS group. Kaplan- Meier curves for OS and DFS according to MSI status of patients are presented in Figure 1.

Also the relation of MSI status and survival in the patients with early stage of disease is evaluated. However, neither OS nor DFS was effected by MSI status in early stage $(p=0.284, p=0.243)$.

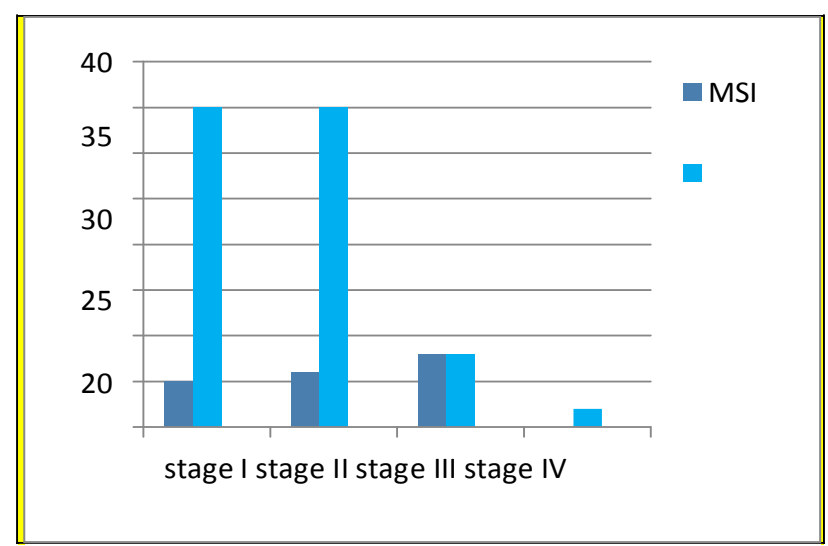

Figure 2: MSI status of patients according to FIGO stage.
MSI is detected in 3 of 12 patients who had recurrence in their follow up (2 lymph node metastasis, 1 distant metastasis). The relationship between MSI and local recurrence $(p=0.586)$ or distant metastasis $(p=$ 0.141 ) was not statistically significant.

$26.3 \%$ of MSI group were stage I, 31\%, $6 \%$ were stage II, $42.1 \%$ were stage III and this relation was statistically significant $(p=0.014)$ (Figure 2$)$. The prevalence of MSI was $13.6 \%$ in early stage and $44.4 \%$ in advanced stage $(p=0.006)$.

A statistically significant difference was seen also between the age and MSI ( $p=0.032)$ (Figure 3). The

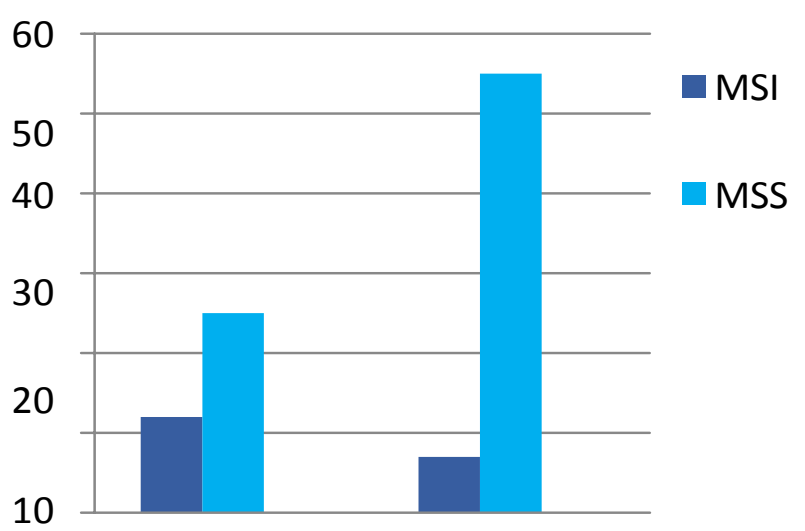

Figure 3: MSI status of patients according to the age.

Table 2: Clinicopathologic features of patients according to MSI status.

\begin{tabular}{|c|c|c|c|c|}
\hline Feature & & MSI & MSS & p value \\
\hline \multirow[t]{4}{*}{ Stage } & 1 & $5(26.3 \%)$ & $35(43.8 \%)$ & 0.014 \\
\hline & II & $6(31.6 \%)$ & $35(43.8 \%)$ & \\
\hline & III & $8(42.1 \%)$ & $8(10 \%)$ & \\
\hline & IV & $0(0 \%)$ & $2(2.5 \%)$ & \\
\hline \multirow[t]{2}{*}{ Stage } & I, II & $11(57.9 \%)$ & $70(87.5 \%)$ & 0.006 \\
\hline & III,IV & $8(42.1 \%)$ & $10(12.5 \%)$ & \\
\hline \multirow[t]{2}{*}{ Myometrial invasion } & $<1 / 2$ & $9(47.4 \%)$ & $42(52.5 \%)$ & 0.883 \\
\hline & $\geq 1 / 2$ & $10(52.6 \%)$ & $38(47.5 \%)$ & \\
\hline \multirow{2}{*}{$\begin{array}{l}\text { Low uterine Segment } \\
+\end{array}$} & No & $9(47.4 \%)$ & $37(46.3 \%)$ & 1.000 \\
\hline & Yes & $10(52.6 \%)$ & $43(53.8 \%)$ & \\
\hline \multirow[t]{2}{*}{ LVi } & No & $9(50 \%)$ & $57(71.3 \%)$ & 0.145 \\
\hline & Yes & $9(50 \%)$ & $23(28.8 \%)$ & \\
\hline \multirow[t]{3}{*}{ Grade } & 1 & $2(10.5 \%)$ & $24(30.4 \%)$ & 0.080 \\
\hline & 2 & $13(68.4 \%)$ & $32(40.5 \%)$ & \\
\hline & 3 & $4(21.1 \%)$ & $23(29.1 \%)$ & \\
\hline \multirow[t]{2}{*}{ Lymph node + } & No & $12(63.2 \%)$ & $60(89.6 \%)$ & 0.012 \\
\hline & Yes & $7(36.8 \%)$ & $7(10.4 \%)$ & \\
\hline \multirow[t]{2}{*}{ Age } & $<60$ & $12(63.2 \%)$ & $26(32.5 \%)$ & 0.027 \\
\hline & $\geq 60$ & $7(36.8 \%)$ & $54(67.5 \%)$ & \\
\hline \multirow[t]{2}{*}{ Local recurrence } & No & $17(89.4 \%)$ & $73(91.2 \%)$ & 0.586 \\
\hline & Yes & $2(10.5 \%)$ & $7(8.7 \%)$ & \\
\hline \multirow[t]{2}{*}{ Distant recurrence } & No & $18(94.7 \%)$ & $78(97.5 \%)$ & 0.141 \\
\hline & Yes & $1(5.2 \%)$ & $2(2.5 \%)$ & \\
\hline
\end{tabular}


mean age was 58.6 years in MSI group, while 63.7 years in MSS group.

The presence of lymph node involvement was statistically significant in patients for MSI status ( $p=$ 0.012). In MSI group, lymph node involvement ratio was $36.8 \%$, while in MSS group it was just $10.4 \%$ (Figure 3$)$.

There was no statistically significant difference of grade $(p=0.080)$, lymphovascular invasion $(p=0.145)$, vascular invasion ( $p=0.303$ ), lower uterine segment involvement $(p=1.000)$ or myometrial invasion $(p=$ 0.883 ) according to MSI status of patients. However, it was noted that MSI-grade relation was close to statistical significance $(p=0.080)$.

In multivariant analysis, age was seen as a protective factor for MSI. The created regression model was statistically significant $(p=0.005)$. Results of the analysis according to variables are presented in Table 3.

\section{Discussion}

There are conflicting results in the literature on the clinicopathologic role of MSI in EC, but several studies have shown that MSI is prognostic to early stage endometrioid EC. These different results might be related due to cohort differences, insufficient statistical power, technical limitations, and evaluation of different histopathologic subtypes together (135). There is a difference in MSI frequency among histopathologic subtypes of EC in studies. The incidence of MSI is 25$30 \%$ in endometrioid EC, $0-14 \%$ in non-endometrioid EC and below $5 \%$ in carcinosarcomas $(145,146,147)$. Also some studies have used non-standard methods that are different from the panel recommended by $\mathrm{NCl}$ for MSI analysis, $(135,144)$.

In a multicenter study performed with early and advanced stage type $1 \mathrm{EC}$ patients, MSI was found strongly associated with high grade ( $p<0.0001)$, but not to have prognostic value (135). Kanopiene, et al. also has reached the conclusion that MSI has no effect on survival. However, similar to our study, they reported that survival time in MSI group was shorter than in MSS group (146) [6].

Mackay, et al. reported that MSI was frequent in early stage cases and MSI had poor prognostic effect in this group $(p<0.0001)$ [7]. Similarly, Steinbakk, et al. reported that MSI has prognostic value only for stage 1 cases in endometrioid adenocarcinoma $(p=0.005)$ [8]. In addition, there was a statistically significant correlation between MSI and grade $(p=0.03)$ in this study [9]. Also in a study of Steinbakk, et al. concluded that low p21 and high survive in expression are poor prognostic indicators in stage I endometrioid adenocarcinoma, especially in MSI presence $[10,11]$.

In our study, MSI was found more frequent in advanced stage endometrioid EC statistically significant. Honore, et al. [12] reported as the same in a cohort study (148). Arabi, et al. also reported a relationship between MSI and advanced disease in 119 high-grade EC patients (57 type I, 62 type II) ( $p=0.039)$ [2]. But they could not show that the MSI has an effect on survival $(p=0.70)$. It is noteworthy that survival in the 3 markers negative MSI group, was statistically significantly worse $(p=0.04)$, and that these cases had a mortality rate of 13.2 times higher, even though the MSI definition and analysis method differed from the $\mathrm{NCl}$ recommendation in this study [2].

There was no statistically significant relationship between $\mathrm{MSI}$ and vascular invasion in the study of Arabi, et al. ( $p=0.19)$ [2]. But in 2009, Bilbao, at al. reported that MSI is related to vascular invasion $(p=$ $0.009)$ and advanced stage $(p=0.04)$ [8]. It is also noted that MSI has an independent predictive value for DFS and local DFS in early stage $(p=0.044)$, suggesting that MSI may have a predictive role in radiotherapy response in this group [6]. Bilbao, et al. also published another study in 2014 that evaluates MSI-ploidy relation in EC. They reported that all MSI tumors were diploid and $14 \%$ of MSS tumors were aneuploid [13]. There was no effect of MSI on DFS or (DSS) disease specific survival. However, it was noted that MSI presence worsened DFS $(p=0.02)$ and DSS $(p=0.02)$ when only diploid tumor group was analyzed (149).

As similar as our study, Steinbakk, et al. did not find a relationship between myometrial invasion depth and MSI ( $p=0.94)$ [8]. But in differently, Arabi, et al. reported that there is a statistically significant relationship between myometrial invasion depth and MSI $(p=0.04)$ [2].

The PORTEC study group also investigated whether the use of molecular genetic alterations in early stage endometrial cancer in combination with traditional prognostic factors was more effective in determining

Table 3: Results of multivariant analysis.

\begin{tabular}{|l|l|l|l|l|}
\hline Variable & OR & Lower (\%95 Cl) & Upper (\%95 Cl) & p value \\
\hline Lymph node involvement & 2.8 & 0.307 & 26.849 & 0.355 \\
\hline Advanced stage & 71 & & & 0.483 \\
\hline Age $\geq 60$ & 2.1 & 0.260 & 17218 & 0.024 \\
\hline & 16 & & & 0.842 \\
\hline
\end{tabular}


risk, and published the results on the internet in March 2016 [14]. In this study, which included long-term results of two large randomized trials (PORTEC I and II), cases divided into 4 subgroups which were defined in TCGA (The Cancer Genome Atlas): p53-mutant (9\%), MSI (26\%), POLE-mutant (POLE exonuclease domain hotspot mutation) (6\%) VE NSMP (no specific molecular profile) (59\%). It was emphasized that p53 mutation was associated with poor prognosis, the effect of MSI and NSMP was moderate, and POLE mutation was associated with good prognosis. Distant recurrence and disease-related death rates were found to be similar among the four groups. However, in the final analysis, excluding good prognostic and poor prognostic factors, MSI was found to be associated with OS and distant recurrence. MSS has been reported to be associated with better prognosis, such as the POLE mutation. In conclusion, it has been emphasized that prognostic molecular changes in early stage EC integrated with already used clinicopathologic factors provide a stronger risk assessment (150) [14].

\section{Conclusion}

In this study, there was no statistically significant effect of MSI on OS ( $p=0.404)$ or on DFS ( $p=0.407)$. But this may be due to the small number of patients and the retrospective design of the study.

MSI is a molecular alteration that occurs at the beginning of carcinogenesis. The presence of MSI in atypical endometrial hyperplasia also supports this view. In the advanced stage of disease, the accumulation of many genetic alterations takes role. So this may explain why that MSI has a prognostic value in early stage and has a limited role in advanced stage EC.

As known, MSI is a good prognostic factor in gastric and colonic cancers. Because of chemotherapy is a frequently used treatment regimen in these cancers, as opposed to early stage EC, it is possible that MSI may be an indicator for chemosensitivity.

MSI in EC appears to contribute to predicting prognosis, especially in early stage disease. As noted in the PORTEC study, it may be more effective to combine MSI and other prognostic molecular alterations with traditional prognostic factors. Further study is needed to fully assess the prognostic value of MSI in EC.

\section{References}

1. Stewart CJ, Amanuel B, Grieu F, Carrello A, lacopetta B (2010) KRAS mutation and microsatellite instability in endometrial adenocarcinomas showing MELF-type myometrial invasion. J Clin Pathol 63: 604-608.

2. Arabi H, Guan H, Kumar S, Cote M, Bandyopadhyay S, et al. (2009) Impact of microsatellite instability (MSI) on survival in high grade endometrial carcinoma. Gynecol Oncol 113: 153-158.

3. Descamps P, Calais G, Moire C, Bertrand P, Castiel M, et al. (1997) Predictors of distant recurrence in clinical stage I or II endometrial carcinoma treated by combination surgical and radiation therapy. Gynecol Oncol 64: 54-58.

4. Bilbao C, Ramírez R, Rodríguez G, Falcón O, León L, et al. (2010) Double strand break repair components are frequent targets of microsatellite instability in endometrial cancer. Eur J Cancer 46: 2821-2827.

5. Zighelboim I, Goodfellow JF, Gao F, Gibb RK, Powell MA, et al. (2007) Microsatellite instability and epigenetic Inactivation of MLH1 and outcome of patients with endometrial carcinomas of the endometrioid type. J Clin Oncol 25: 2042-2048.

6. Kanopiene D, Smailyte G, Vidugiriene J, Bacher J (2014) Impact of microsatellite instability on survival of endometrial cancer patients. Medicana 50: 216-221.

7. Mackay HJ, Gallinger S, Tsao MS, McLachlin CM, Tu D, et al. (2010) Prognostic value of microsatellite instability (MSI) and PTEN expression in women with endometrial cancer: results from studies of the NCIC Clinical Trials Group (NCIC CTG). Eur J Cancer 46: 1365-1373.

8. Steinbakk A, Malpicia A, Sleva A, Gudlaugsson E, Janssen EA, et al. (2010) High frequency microsatellite instability has a prognostic value in endometrial endometrioid adenocarcinoma, but only in FIGO stage 1 cases. Cell Oncol 33: 245-255.

9. Sartori E, Laface B, Gadducci A, Maggino T, Zola P, et al. (2003) Factors influencing survival in endometrial cancer relapsing patients: a Cooperation Task Force (CTF) study. Int J Gynecol Cancer 13: 458-465.

10. Steinbakk A, Malpica A, Slewa A, Skaland I, Gudlaugsson $\mathrm{E}$, et al. (2011) Biomarkers and microsatellite instability analysis of curettings can predict the behavior of FIGO stage I endometrial endometrioid adenocarcinoma. Mod Pathol 24: 1262-1271.

11. Zusterzeel PL, Bekkers RL, Hendriks JC, Neesham DN, Rome RM, et al. (2008) Prognostic factors for recurrence in patients with FIGO stage I and Prognostic factors for recurrence in patients with FIGO stage I and II, intermediate or high risk endometrial cancer. Acta Obstet Gynecol Scand 87: 240-246.

12. Honore LH, Hanson J, Andrew SE (2006) Microsatellite instability in endometrioid endometrial carcinoma: correlation with clinically relevant pathologic varibles. Int $\mathrm{J}$ Gynecol Cancer 16: 1386-1392.

13. Bilbao-Sieyro C, Ramirez R, Rodriquez Gonzales G, Falcón O, León L, et al. (2014) Microsatelite instability and ploidy status define three categories with distinctive prognostic impact in endometrioid endometrial cancer. Oncotarget 5: 6206-6217.

14. Stello E, Nout RA, Osse EM, Jürgenliemk-Schulz IJ, Jobsen $\mathrm{JJ}$, et al. (2016) Improved risk assessment by integrating molecular and clinicopathological factors in early-stage endometrial cancer-combined analysis of the PORTEC cohorts. Clin Cancer Res 22: 4215-4224. 